\title{
WILEY-VCH
}

DOI: $10.1002 /(($ please add manuscript number $))$

Article type: Communication

\section{Engineering DNA binding sites to assemble and tune plasmonic nanostructures}

Alasdair W. Clark*, David G. Thompson, Duncan Graham, Jonathan M. Cooper

Dr. A.W. Clark, Prof. J.M.

Biomedical Engineering Research Division, School of Engineering, University of Glasgow, Glasgow, UK, G12 8LT

E-mail: Alasdair.clark@glasgow.ac.uk

Dr. D.G. Thompson, Prof. D. Graham

Centre for Molecular Nanometrology, WestCHEM, Department of Pure and Applied

Chemistry, University of Strathclyde, Glasgow, UK, G1 1XL

Keywords: Plasmonics, Surface Enhanced Raman Spectroscopy, DNA, Biosensor, Molecular Assembly

Nanoplasmonics enables the confinement and manipulation of electromagnetic waves around nanometallic structures, providing an unprecedented degree of optical control beyond the diffraction limit. As a result, engineered construction of nanoplasmonic structures has led to the creation of a host of nanoscale materials with photonic properties that are not found in nature. ${ }^{[1-2]}$ Harnessing the power of complex nanophotonic assemblies has been heralded as a means to improve upon the all-electrical circuitry currently at the core of modern computing architecture ${ }^{[3-8]}$ and has already had a significant impact within the life sciences; where the nanoscale photonic control enabled by localised surface plasmon resonance (LSPR) ${ }^{[9]}$ has extended the sensitivity of molecular detection systems in a wide variety of applications, encompassing cell biology, ${ }^{[10-12]}$ imaging, ${ }^{[13-14]}$ sensing, ${ }^{[15-17]}$ and diagnostics. ${ }^{[18]}$ Key to the advancement of these fields are reliable strategies for the fabrication of high-resolution nanoscale materials and functional devices. To date, production has largely followed different paths; top-down nanolithography ${ }^{[19-21]}$ and molecularly-mediated nanoparticle assembly, ${ }^{[22-24]}$ with limited integration of the two approaches. While having the potential to outstrip the resolution constraints of top-down techniques, controlling the molecular assembly of 


\section{WILEY-VCH}

nanoparticle networks on surfaces is sufficiently challenging that its use in surface-based plasmonic applications has been limited. Here, we integrate these ideas, engineering DNA binding sites to assemble and tune plasmonic surface structures. Using DNA-directed placement of single nanoparticles within engineered nanophotonic structures, we create coupled plasmonic bowties with molecularly controlled photonic properties. Acting to both define the geometry of the sensor and specifically tune its optical response, the DNA trigger reconfigures the plasmonic function of the bowtie, creating two intense plasmonic hotspots at an engineered frequency. In doing so, we build a sensor capable of recording individual DNA-nanoparticle binding events, using a coupled nanophotonic bowtie that is triggered by the same DNA hybridisation it is designed to detect.

Acting as antennae for incident light radiation, the plasmonic resonances inherent to metallic nanoparticles generate intense, highly localised electric fields which are the key component in enhanced vibrational spectroscopies such as SERS (surface enhanced Raman scattering). ${ }^{[25]}$ By aggregating discrete nanoparticles, the field can be increased further and exceptionally large enhancement values can be realised due to interparticle plasmoniccoupling. Controlling these interactions is paramount to harnessing the full potential of these enhanced vibrational spectroscopies. Molecularly driven aggregation in solutions, where particles cluster due to binding events, can offer a degree of interparticle spatial control to increase enhancement, ${ }^{[26-29]}$ yet is limited in scope and requires large amounts nanoparticles to be effective. By translating techniques such as these to surfaces, there is the potential to obtain positional control of individual binding events by strictly regulating the molecular surface pattern. In doing so, the position of single nanoparticle binding reactions can be engineered, ${ }^{[30]}$ opening the door for the creation of complex plasmonic surfaces that actively react to their molecular environment. 


\section{WILEY-VCH}

In this paper, we create bowtie nanostructures with structural and photonic properties which are altered due to engineered nanoparticle binding events. Using DNA-directed assembly, individual nanoparticles are bound within the gaps of nanophotonic bowtie structures (gold triangular nano-prism dimers) fabricated using electron-beam lithography, Figure 1. By using molecular binding as a building block in the construction the sensor, we can create gaps that would difficult to produce lithographically, while also ensuring those gaps are generated by the binding event of interest. Acting as a plasmonic-bridge, the nanoparticle couples plasmon of the three discrete objects, shifting their LSPR to a chosen laser wavelength while creating two well-defined electromagnetic "hot-spots" as tightly confined areas of plasmonic enhancement. Hot-spot generation through nanoparticle aggregation is the fundamental technique that underpins the majority of SERS research. ${ }^{[26-27,31-33]}$ Indeed, the interest in molecularly assembled nanoparticle networks, both for use in SERS and as biologically activated building-blocks for bottom-up fabrication of photonic surfaces, has recently seen rapid growth. ${ }^{[24,34-42]}$ In our implementation, the fabricated nano-prisms form an incomplete, non-functional sensor, tuned and activated only when coupled by single DNA-nanoparticle binding. Using DNA-hybridisation to alter the photonic and geometric properties of the nanostructures is particularly appealing as it provides an almost unlimited level of selectivity and programmability for multi-species construction.

The bowties were fabricated on a glass substrate using a combination of electron-beam lithography and electron-beam metal evaporation, Figure 1 (see Experimental Section for full fabrication details). The equilateral Au triangular prisms had axes of $65 \mathrm{~nm}$ and a thickness of $27 \mathrm{~nm}$. The gap between each dimer was designed to be $44 \mathrm{~nm}$. Acting as antennae for incident light radiation, the structures generate large electric-fields at their opposing tips due to the plasmonic focusing effect of the individual prisms and the inter-structure field-coupling which takes place when these structures are excited in-phase. ${ }^{[20,43-44]}$ Patterning this small 


\section{WILEY-VCH}

inter-structure area with DNA (using an electron-beam defined polymer mask aligned to the original structures to enable nanoscale deposition of 3-glycidyloxypropyltrimethoxysilane followed by an amine-modified oligonucleotide, 5' amine (HEG) 3 TACGAGTTGAGA) allowed us to capture single $40 \mathrm{~nm}$ diameter nanoparticles, conjugated with a complimentary oligonucleotide (5' $\mathrm{SH}$ (HEG) ${ }_{3}$ TCTCAACTCGTA), within the gap (see the Experimental Section for nanoparticle and DNA binding protocols).

Taking advantage of the precision allowed by nanolithography, we are able to largely dictate the location of the binding events so that, in the majority of cases, only a single nanoparticle can bind in a specifically chosen area ${ }^{[30]}$ Placing the nanoparticles within a dimer structure has the advantage of increasing the chance of forming small gaps which in turn create strong plasmon hotspots (when compared to placing a particle next to a single surface feature). In our experiments, the particle location within the dimer varies from being roughly centred (creating gaps of $\sim 2 \mathrm{~nm}$ on either side), to touching one of the prism tips (creating a gap of $\sim 4 \mathrm{~nm}$ to the opposing prism). Figure 2 shows the precision that can be achieved using this technique. Approximately $73 \%$ of the total bowties sampled had a nanoparticle occupying their centre, with $>90 \%$ of these features displaying single occupancy ( $\mathrm{n}=639$ features), while non-specific binding to areas of the glass surface was low (approximately 80 non-specifically bound nanoparticles in a sample area of $6.17 \times 10^{-11} \mathrm{~m}^{2}$ ). Although both DNA and amine groups have affinity for $\mathrm{Au}$, the lack of significant nanoparticle binding to areas around the structures other than the intended centre spots shows that non-specific DNA adsorption onto the prisms is also low.

In order for the sensor to operate in the most efficient manner, the LSPR of the coupled bowtie was designed so that it corresponded to the desired input laser wavelength of $633 \mathrm{~nm}$. Consequently, the resonances of the discrete device elements (the dimers and the 


\section{WILEY-VCH}

nanoparticle) do not conform to this requirement, experiencing LSPR at $610 \mathrm{~nm}$ and $520 \mathrm{~nm}$ respectively. Electromagnetic coupling of these nanostructures through DNA-binding has two important effects; it shifts the LSPR of the system to a longer wavelength ${ }^{[45-46]}$ and, most importantly for a SERS device, creates two hotspots of electric-field confinement at the small junctions between the tips of the prisms and the nanoparticle. ${ }^{[4]}$ Extinction spectroscopy, Figure 3(a)(i), shows the LSPR consequence of the plasmonic coupling, the peak red-shifting from 610 to $630 \mathrm{~nm}$ after DNA hybridization.

Finite element simulations (performed using COMSOL) were used to both optimize the LSPR frequency of the design, as well as the electric-field hotspot localization around the coupled bowtie, Figure 3(a)(ii) and 3(b) (see Experimental Section for simulation details). Figure 3(b) shows the simulated electric-field enhancement experienced by a complete and incomplete bowtie at resonance, as well as an isolated nanoparticle, at their respective resonance wavelengths. The incomplete bowtie structure, its tips separated by $\sim 44 \mathrm{~nm}$, shows weak tip-tip coupling at its LSPR peak and displays a maximum electric field enhancement of 30x inside this gap. Upon nanoparticle binding, the localized electric field generated at the tips is significantly greater, and although there is some slight variation in the location of bound particles, not having the particles bind perfectly in the centre of the dimer may be advantageous for SERS hotspot generation. The FEM calculated fields show enhancements of $>1700$ when the particle is located exactly in the centre of the dimer, and $>18000$ for the instances when the nanoparticle is touching one of the prisms (Supporting Information Figure S5). In both cases, the important result of the binding is the generation of two gaps that result in large field enhancements. This requirement is met as long as the nanoparticle binds somewhere between the tips. 


\section{WILEY-VCH}

To demonstrate the applicability of this new strategy for ultrasensitive biosensing, we now apply our DNA-triggered bowtie structures to SERS, ${ }^{[26-27,32]}$ using the assembly and activation of the bowtie as a means to sense DNA hybridisation events. In order to determine the SERS impact of the circuit's engineered hotspots, arrays of oligonucleotides with various periodicities $(300,750$ and $2000 \mathrm{~nm})$ were prepared on glass surfaces, with and without bowtie structures present (Supplementary Information Figure S1). Nanoparticles were then bound to these patterns via DNA hybridisation. The nanoparticles were modified in equal measure with DNA complementary to that patterned on the surface and the Raman reporter dye, malachite green isothiocyanate $(\mathrm{MG})$. In an array of such devices we are able to control the number of binding events taking place, and thus the location of each hotspot. This addresses a significant challenge when working with nanoparticles for SERS; the targeted localization and LSPR tuning of hotspots. ${ }^{[48-49]}$

As demonstrated in Figure 3, precise y-axis alignment of the bowtie tips and the bound nanoparticle ensures that the hotspots engulf large portions of the nanoparticle surface area, where, crucially, the Raman reporter dye is located, therefore maximizing the efficiency of the Raman scattering from each bowtie. Used in conjunction with a 100x, 0.75 NA objective, the focused $633 \mathrm{~nm}$ laser spot had a power of $1.8 \mathrm{~mW}$ (measured at the objective) and a minimum diameter of $1.03 \mu \mathrm{m}$ when focused on the surface. This corresponds to approximately 9 devices, and therefore 18 hotspots, under interrogation when the structures have a periodicity of $300 \mathrm{~nm}$. Since we can position each binding event, there is a known number of hotspots at defined locations. Figure 4 compares SERS spectra from the $300 \mathrm{~nm}$ period arrays, with and without the nanoparticle integrated within the bowtie. Taking the strong Raman peak associated with MG's C-C stretch mode for comparison, ${ }^{[50]}$ which appears in our measurements at $1605 \mathrm{~cm}^{-1}$, the isolated nanoparticles show an average intensity of $>100$ counts for a 5 second acquisition time, whereas the completed bowties, with the same 


\section{WILEY-VCH}

periodicity, show an average of $>23300$ counts, a SERS signal which is $>210 x$ larger than the signal seen from the isolated nanoparticles.

The asymmetric arrangement of the plasmonic bowtie dictates that it is only functional for one input polarization. ${ }^{[51-53]}$ When excited in phase, the charges at each interface of the 3element bowtie are opposed, creating an attractive force which couples each element's electron-cloud across the entire bowtie. We use this dependence on the polarization of the excitation as an additional level of control, providing "on" and "off" states for the device. When the structure is rotated through 90 degrees with respect to the laser, the plasmon of the bowtie is effectively turned off (Figure 5(a)). The charges at the tips of each prism and the opposing surface of the nanoparticle are then identical, so no plasmon coupling takes place across the gap (in the case of a particle centered within the dimer, maximum field values drop from above 1700x in the "on" position to below 10x in the "off" position). This large drop in hotspot enhancement caused by the incorrect input condition directly impacts the Raman output of the device. The SERS signal collected from the $300 \mathrm{~nm}$ period array in either arrangement is shown in Figure 5(b). In the "on" condition $>4200$ counts per second were collected, whereas for the "off" condition this value dropped to approximately 400 counts per second.

The ability to record single biomolecular binding events represents the pinnacle of sensitivity in biosensing and diagnostics. Here, we show that our devices are capable of recording individual DNA-nanoparticle binding events via SERS. In order to demonstrate this, arrays of patterned DNA were prepared with various periodicities, with and without bowtie structures present (information on the isolated nanoparticle binding can be found in Figure S1 of the Supporting Information). The arrays had a periodicity of 300, 750 and 2000 $\mathrm{nm}$ respectively. Given the minimum laser spot size mentioned previously, this relates to 


\section{WILEY-VCH}

approximately 9.3 and 1.5 bowties being driven by the laser for the 300 and $750 \mathrm{~nm}$ arrays respectively, and only a single bowtie in the case of the $2000 \mathrm{~nm}$ array. Knowing the exact location of each sensor allows us to pinpoint individual binding sites with the Raman microscope using the same alignment marks which make the fabrication of the system possible.

Figure 6(a) shows a comparison of Raman scattering from the $1605 \mathrm{~cm}^{-1}$ peak for all 3 arrays, with and without bowties (represented by the black and red bars, respectively). In all cases the SERS signal from the bowties was significantly larger than that of the isolated nanoparticles for an identical number of binding events. Notably, for a single, isolated nanoparticle binding event with no bowtie circuit present, no SERS signal could be recorded using any acquisition time. The field generated by the relatively weak LSPR of the single nanoparticle is not large enough to enhance the Raman scattering from such a small number of reporter molecules. However, as illustrated by Figure 3, the two hotspots generated by the nanoparticle binding within a bowtie creates electric field enhancements far exceeding those seen for an isolated nanoparticle. Figure 6(b) shows the Raman scattered output is sufficiently large as to record a SERS signal from a single nanoparticle binding event. Employing DNA hybridization is a key aspect in the sensor's functionality, and enables us to record single nanoparticle binding events without using advanced microscopies such as SEM, or inferring them through statistical analysis.

In summary, we have demonstrated new plasmonic SERS sensors which incorporate single DNA-nanoparticle binding events into their construction and activation; using DNA hybridisation to simultaneously generate the SERS hotspots and tune the plasmonic frequency of the sensor. In doing so, we demonstrate a nanophotonic Raman sensor which only works when a nanoparticle is anchored within its geometry by DNA hybridization it seeks to 


\section{WILEY-VCH}

measure. Although the use of such a DNA binding strategy for real-world diagnostics may prove limited (due to requiring pre-labelling of single DNA strands), using DNA as the nanoparticle placement device also opens up the sensing capabilities of the bowties to include other biologically relevant moieties such as proteins and toxins through the use of aptamers. Not only does this technique represent a new route for controlled, post-fabrication tuning of plasmonic surfaces and structures, but has enabled us to build an extremely sensitive, reproducible platform for monitoring the DNA-mediated binding of single nanoparticles. This generic platform can be applied to detecting other molecular interactions, being most applicable for measuring biological interactions between species where sensitivity is an issue - for example, in determining protein-protein interactions where an equivalent of PCR amplification does not exist.

\section{Experimental Section}

\section{DNA-nanoparticle Synthesis:}

Oligonucleotide sequences were supplied by ATD Bio, UK. Malachite Green isothiocyanate was purchased from Invitrogen,UK. All other chemicals were supplied by Sigma Aldrich, UK.

Gold nanoparticles were synthesised using a modified Frens ${ }^{[54]}$ method. Briefly, $57.5 \mathrm{mg}$ of sodium tetrachloroaurate was dissolved in $500 \mathrm{~mL}$ of doubly distilled deionised water and heated until boiling. $60.5 \mathrm{mg}$ of trisodium citrate dihydrate dissolved in $10 \mathrm{~mL}$ of doubly distilled water was then added and the solution boiled for a further 15 minutes. The sol was then allowed to cool and the concentration determined using UV-Vis spectrometry.

DNA-nanoparticle conjugates were made using the previously reported method ${ }^{[55]} 5$ nmoles of thiol-modified oligonucleotide 5' $\mathrm{SH}$ (HEG) $)_{3}$ TCTCAACTCGTA was added to $1.5 \mathrm{~mL}$ of the prepared gold nanoparticle sol and salt-aged as previously reported ${ }^{[56]}$ before being 


\section{WILEY-VCH}

centrifuged (5000 rpm, $20 \mathrm{~min}$ ) and resuspended in $1 \mathrm{~mL} 0.3 \mathrm{M}$ phosphate-buffered saline (PBS) ph 7.4. Malachite green isothiocyanate $(500 \mu \mathrm{L}, 1 \mu \mathrm{M})$ was added to the gold nanoparticle solution and allowed to equilibrate overnight in the dark. The DNA-nanoparticle conjugates were then centrifuged and resuspended in 0.3 M PBS ph 7.4.

\section{Plasmonic Bowtie fabrication:}

The plasmonic bowtie structures were fabricated by standard electron-beam lithography (EBL), metal evaporation and resist lift-off procedures. Figure 1(a) shows a schematic of the of the details fabrication process. A PMMA bilayer (2010 PMMA followed by 2041 PMMA is used to create an undercut profile after exposure and development, which aids resist liftoff), with a final thickness of approximately 90nm, was spun onto a $500 \mu \mathrm{m}$ thick Pyrex substrate and exposed to a $100 \mathrm{kV}$ electron-beam using a Vistec VB6 UHR EWF electronbeam lithographer. Written in this lithography step were the dimer arrays, along with a series of alignment marks to enable a second pattern to be aligned to this first one. The resist was developed using 2.5:1, IPA:MIBK for 45 seconds at $23^{\circ} \mathrm{C}$. A $2 \mathrm{~nm}$ adhesion layer of Ti was evaporated onto the sample, followed immediately by a $25 \mathrm{~nm}$ layer of Au. The sample was then soaked in a $50{ }^{\circ} \mathrm{C}$ acetone bath for one hour to lift-off the remaining PMMA.

In order to fabricate the windows, which allowed the surface to be selectively patterned with DNA, the sample was recoated with an identical PMMA bilayer and the windows were defined by the electron-beam lithography tool (designed as $40 \mathrm{~nm}$ squares which, upon development created $40 \mathrm{~nm}$ squares with rounded edges). Au alignment marks patterned in the previous step ensured that each $40 \mathrm{~nm}$ window was positioned in the centre of the each dimer. The windows were developed using 2.5:1, IPA:MIBK for 45 seconds at $23^{\circ} \mathrm{C}$.

DNA binding of individual nanoparticles to the surface: 


\section{WILEY-VCH}

The glass surface below each $40 \mathrm{~nm}$ window was modified with 3glycidyloxypropyltrimethoxysilane (Sigma-Aldrich) by placing the sample in a $4 \%$ solution, in ethanol, for 4 hours. After rinsing with ethanol, the samples were dried with nitrogen and placed in a $90{ }^{\circ} \mathrm{C}$ oven for 1 hour. The samples were then soaked in $50{ }^{\circ} \mathrm{C}$ acetone bath for one hour to lift-off the remaining PMMA, leaving behind a glass surface modified with $40 \mathrm{~nm}$ patches of epoxy-silane between each Au dimer.

$10 \mu 1$ of $200 \mathrm{nM}$ amine-modified oligonucleotide $5^{\prime}$ amine (HEG) $)_{3}$ TACGAGTTGAGA was spotted onto the sample and incubated at $4{ }^{\circ} \mathrm{C}$ for 2 hours. The sample was washed with PBS (phosphate buffered saline, $\mathrm{pH} 7$ ), dried with nitrogen and then immediately spotted with 10 $\mu 1$ of the DNA-nanoparticle conjugate (5' SH (HEG) $)_{3}$ TCTCAACTCGTA). The sample was left to incubate for 18 hours at $4{ }^{\circ} \mathrm{C}$ before being washed thoroughly, in turn with PBS and distilled water.

Given the oligonucleotide size and the binding chemistry used, the number of DNA strands estimated to be attached to each nanoparticle was $\sim 1550^{[57]}$, while the number of strands attached to the $40 \times 40 \mathrm{~nm}$ surface was estimated, based on our immobilisation method, to be $\sim 20 .^{[58-59]}$ The relative excess of DNA on the particle compared to the surface aids the chances of successful hybridisation. The exact number of bound molecules hybridising is difficult to estimate as it will be influenced by, among other things, particle curvature at the point of contact, crowding from other adjacent molecules, and exact density of the DNA on both the surface and the particle at the point of contact.

\section{Optical characterisation:}

The plasmonic characteristics of each sample were measured using a Shimadzu UV3101PC absorption spectrometer. A Thor Labs linear polariser was used to orientate the electric field of the exciting light along the long-axis of the bowties. The experimental range was $400-800$ nm. 


\section{WILEY-VCH}

\section{Finite Element Simulation:}

The plasmonic response of the system was modelled using COMSOL Multiphysics, a finite element method (FEM) package. Using this method both extinction spectra and localised electric field distributions were calculated for the isolated nanoparticles, the incomplete bowties and the bowties completed by a nanoparticle after DNA hybridisation. The simulation geometries were taken from the SEM measurements of the experimental structures. The nanoparticle was placed within the simulations at a separation of $2 \mathrm{~nm}$ from the tip of each prism. The simulation space was delimited by perfectly matched layers to prevent unwanted reflections. The incident electric field value, E0, was set to 1 , and represented by either an $\mathrm{x}$ or $\mathrm{y}$-polarised wave propagating in the z-direction. The simulation space consisted of a $600 \mathrm{~nm}$ radius sphere containing $>105^{\prime} 000$ mesh elements. Johnson and Christy's dielectric function of gold ${ }^{[60]}$ was used to model the frequency dispersive response of the scatterer's electrical permittivity.

\section{Raman measurements:}

Raman measurements were performed using a Horiba Jobin Yvon HR800UV Raman spectrometer in conjunction with an Olympus IX71 inverted microscope. The power of the $633 \mathrm{~nm} \mathrm{HeNe}$ laser used in these experiments was measured at the objective to be $1.8 \mathrm{~mW}$ when used in conjunction with a 100x, 0.75 NA objective lens.

\section{Supporting Information}

Supporting Information is available from the Wiley Online Library or from the author.

\section{Acknowledgements}




\section{WILEY-VCH}

The work was supported by the Royal Academy of Engineering (grant number 10216/103). Device fabrication was carried out in the James Watt Nanofabrication Centre at the University of Glasgow. The authors would like to thank Steven Neale and Julien Reboud for their help preparing the manuscript.

Received: ((will be filled in by the editorial staff))

Revised: ((will be filled in by the editorial staff)) Published online: ((will be filled in by the editorial staff))

[1] J. B. Pendry, D. Schurig, D. R. Smith, Science 2006, 312, 1780.

[2] V. M. Shalaev, Nat. Photonics 2007, 1, 41.

[3] A. L. Falk, F. H. L. Koppens, C. L. Yu, K. Kang, N. D. Snapp, A. V. Akimov, M. H. Jo, M. D. Lukin, H. Park, Nature Physics 2009, 5, 475.

[4] D. K. Gramotnev, S. I. Bozhevolnyi, Nat. Photonics 2010, 4, 83.

[5] S. Lal, J. H. Hafner, N. J. Halas, S. Link, P. Nordlander, Accounts of Chemical Research 2012, 45, 1887.

[6] D. Rossouw, G. A. Botton, Physical Review Letters 2013, 110.

[7] V. J. Sorger, R. F. Oulton, R. M. Ma, X. Zhang, Mrs Bulletin 2012, 37, 728.

[8] H. Wei, Z. X. Wang, X. R. Tian, M. Kall, H. X. Xu, Nature Communications 2011, 2.

[9] C. Bohren, D. Huffman, Absorption and Scattering of Light by Small Particles, John Wiley \& Sons, 1983.

[10] N. M. S. Sirimuthu, C. D. Syme, J. M. Cooper, Anal. Chem. 2010, 82, 7369.

[11] P. K. Jain, X. H. Huang, I. H. El-Sayed, M. A. El-Sayed, Accounts of Chemical Research 2008, 41, 1578.

[12] C. J. Murphy, A. M. Gole, J. W. Stone, P. N. Sisco, A. M. Alkilany, E. C. Goldsmith, S. C. Baxter, Accounts of Chemical Research 2008, 41, 1721. 


\section{WILEY-VCH}

[13] C. J. Murphy, T. K. San, A. M. Gole, C. J. Orendorff, J. X. Gao, L. Gou, S. E. Hunyadi, T. Li, Journal of Physical Chemistry B 2005, 109, 13857.

[14] P. K. Jain, K. S. Lee, I. H. El-Sayed, M. A. El-Sayed, Journal of Physical Chemistry B 2006, 110,7238 .

[15] R. Elghanian, J. J. Storhoff, R. C. Mucic, R. L. Letsinger, C. A. Mirkin, Science 1997, $277,1078$.

[16] J. N. Anker, W. P. Hall, O. Lyandres, N. C. Shah, J. Zhao, R. P. Van Duyne, Nature Materials 2008, 7, 442 .

[17] P. Alivisatos, Nature Biotechnology 2004, 22, 47.

[18] E. Boisselier, D. Astruc, Chemical Society Reviews 2009, 38, 1759.

[19] R. Adato, A. A. Yanik, H. Altug, Nano Lett. 2011, 11, 5219.

[20] N. A. Hatab, C. H. Hsueh, A. L. Gaddis, S. T. Retterer, J. H. Li, G. Eres, Z. Y. Zhang, B. H. Gu, Nano Lett. 2010, 10, 4952.

[21] J. Ye, F. F. Wen, H. Sobhani, J. B. Lassiter, P. Van Dorpe, P. Nordlander, N. J. Halas, Nano Lett. 2012, 12, 1660.

[22] C. A. Mirkin, R. L. Letsinger, R. C. Mucic, J. J. Storhoff, Nature 1996, 382, 607.

[23] D. Nykypanchuk, M. M. Maye, D. van der Lelie, O. Gang, Nature 2008, 451, 549.

[24] A. M. Hung, C. M. Micheel, L. D. Bozano, L. W. Osterbur, G. M. Wallraff, J. N. Cha, Nature Nanotechnology 2010, 5, 121.

[25] A. Campion, P. Kambhampati, Chemical Society Reviews 1998, 27, 241.

[26] L. Guerrini, D. Graham, Chemical Society Reviews 2012, 41, 7085.

[27] D. Graham, D. G. Thompson, W. E. Smith, K. Faulds, Nature Nanotechnology 2008, 3,548 .

[28] G. P. Acuna, F. M. Moeller, P. Holzmeister, S. Beater, B. Lalkens, P. Tinnefeld, Science 2012, 338, 506. 


\section{WILEY-VCH}

[29] D.-K. Lim, K.-S. Jeon, H. M. Kim, J.-M. Nam, Y. D. Suh, Nature Materials 2010, 9, 60.

[30] A. W. Clark, J. M. Cooper, Angewandte Chemie-International Edition 2012, 51, 3562.

[31] Y. H. Zheng, T. Thai, P. Reineck, L. Qiu, Y. M. Guo, U. Bach, Advanced Functional Materials 2013, 23, 1519.

[32] Y. J. Sun, F. G. Xu, Y. Zhang, Y. Shi, Z. W. Wen, Z. Li, Journal of Materials Chemistry 2011, 21, 16675.

[33] G. Braun, S. J. Lee, M. Dante, T.-Q. Nguyen, M. Moskovits, N. Reich, Journal of the American Chemical Society 2007, 129, 6378.

[34] B. Q. Ding, Z. T. Deng, H. Yan, S. Cabrini, R. N. Zuckermann, J. Bokor, Journal of the American Chemical Society 2010, 132, 3248.

[35] A. N. Shipway, E. Katz, I. Willner, Chemphyschem 2000, 1, 18.

[36] J. P. Spatz, V. Z. H. Chan, S. Mossmer, F. M. Kamm, A. Plettl, P. Ziemann, M. Moller, Advanced Materials 2002, 14, 1827.

[37] C. H. Lalander, Y. Zheng, S. Dhuey, S. Cabrini, U. Bach, Acs Nano 2010, 4, 6153.

[38] P. Anstaett, Y. H. Zheng, T. Thai, A. M. Funston, U. Bach, G. Gasser, Angewandte Chemie-International Edition 2013, 52, 4217.

[39] J. W. Zheng, P. E. Constantinou, C. Micheel, A. P. Alivisatos, R. A. Kiehl, N. C. Seeman, Nano Lett. 2006, 6, 1502.

[40] H. Noh, A. M. Hung, C. Choi, J. H. Lee, J. Y. Kim, S. Jin, J. N. Cha, Acs Nano 2009, 3, 2376 .

[41] S. I. Lim, C. J. Zhong, Accounts of Chemical Research 2009, 42, 798.

[42] S. J. Tan, M. J. Campolongo, D. Luo, W. L. Cheng, Nature Nanotechnology 2011, 6, 268.

[43] H. Fischer, O. J. F. Martin, Optics Express 2008, 16, 9144. 


\section{WILEY-VCH}

[44] D. P. Fromm, A. Sundaramurthy, P. J. Schuck, G. Kino, W. E. Moerner, Nano Lett. $\mathbf{2 0 0 4}, 4,957$.

[45] B. Lamprecht, G. Schider, R. T. Lechner, H. Ditlbacher, J. R. Krenn, A. Leitner, F. R. Aussenegg, Physical Review Letters 2000, 84, 4721.

[46] W. Rechberger, A. Hohenau, A. Leitner, J. R. Krenn, B. Lamprecht, F. R. Aussenegg, Optics Communications 2003, 220, 137.

[47] K. L. Kelly, E. Coronado, L. L. Zhao, G. C. Schatz, Journal of Physical Chemistry B 2003, 107, 668 .

[48] F. T. Docherty, P. B. Monaghan, C. J. McHugh, D. Graham, W. E. Smith, J. M. Cooper, Ieee Sensors Journal 2005, 5, 632.

[49] K. Faulds, R. E. Littleford, D. Graham, G. Dent, W. E. Smith, Anal. Chem. 2004, 76, 592.

[50] H. B. Lueck, D. C. Daniel, J. L. McHale, Journal of Raman Spectroscopy 1993, 24, 363.

[51] A. W. Clark, J. M. Cooper, Advanced Materials 2010, 22, 4025.

[52] A. W. Clark, J. M. Cooper, Small 2011, 7, 119.

[53] A. W. Clark, A. Glidle, D. R. S. Cumming, J. M. Cooper, Journal of the American Chemical Society 2009, 131, 17615.

[54] G. Frens, Nature-Physical Science 1973, 241, 20.

[55] L. Barrett, J. A. Dougan, K. Faulds, D. Graham, Nanoscale 2011, 3, 3221.

[56] F. McKenzie, K. Faulds, D. Graham, Small 2007, 3, 1866.

[57] S. J. Hurst, A. K. R. Lytton-Jean, C. A. Mirkin, Anal. Chem. 2006, 78, 8313.

[58] J. B. Lamture, K. L. Beattie, B. E. Burke, M. D. Eggers, D. J. Ehrlich, R. Fowler, M. A. Hollis, B. B. Kosicki, R. K. Reich, S. R. Smith, R. S. Varma, M. E. Hogan, Nucleic Acids Res. 1994, 22, 2121. 


\section{WILEY-VCH}

[59] W. G. Beattie, L. Meng, S. L. Turner, R. S. Varma, D. D. Dao, K. L. Beattie, Mol. Biotechnol. 1995, 4, 213.

[60] P. B. Johnson, R. W. Christy, Phys. Rev. B 1974, 9, 5056. 


\section{WILEY-VCH}
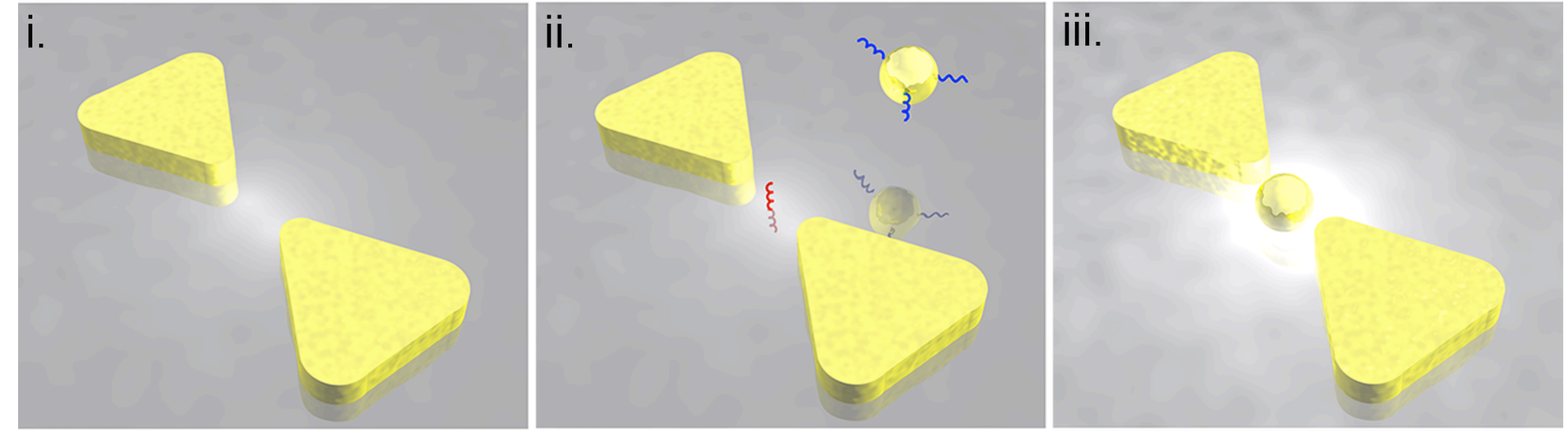

Figure 1. Fabrication schematic of a DNA-activated plasmonic bowtie. (i) Au triangularprism dimers fabricated on a glass substrate. (ii) The dimer gap modified with oligonucleotide enabling it to bind to a single $\mathrm{Au}$ nanoparticle conjugated with a complimentary oligonucleotide. (iii) A coupled plasmonic bowtie, switched on by DNA-hybridization.

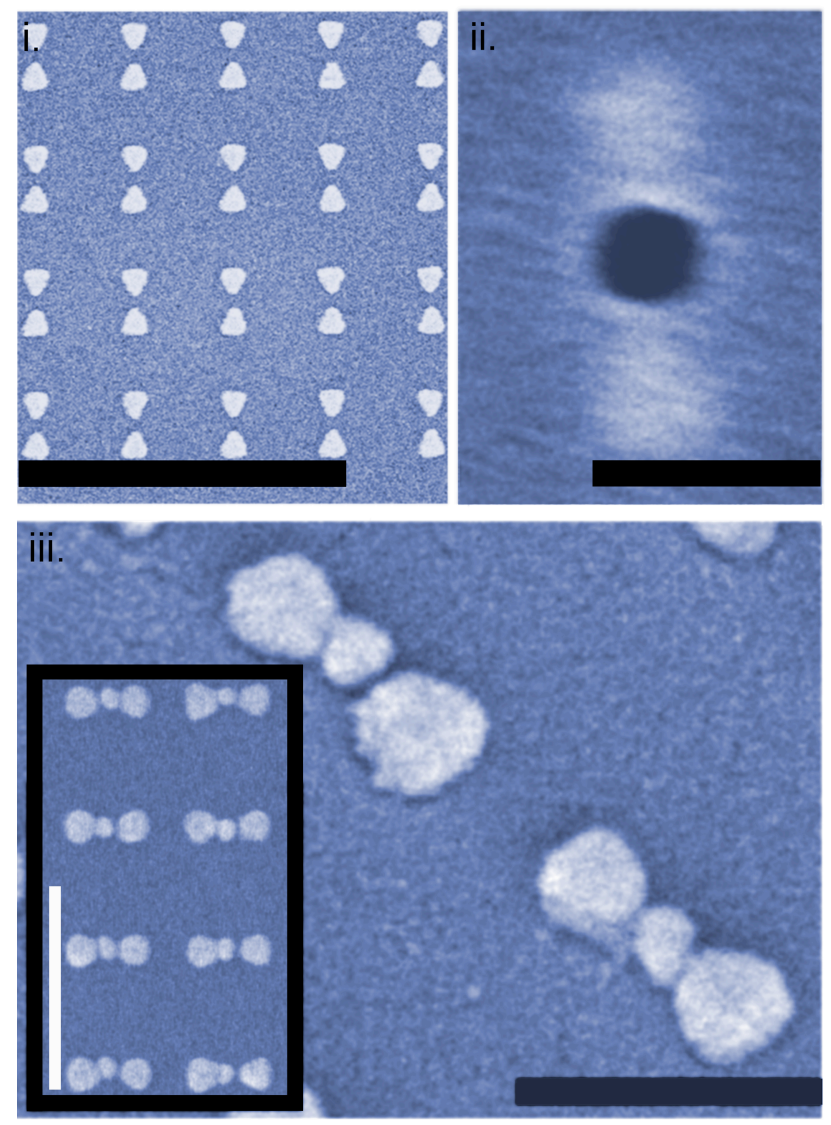

Figure 2. SEM images detailing sensor construction (i) an incomplete bowtie array, (ii) an individual dimer with a $40 \mathrm{~nm}$ polymer window aligned to its gap, (iii) coupled devices completed with the inclusion of a DNA-nanoparticle. The scale bars represent $1 \mu \mathrm{m}, 100 \mathrm{~nm}$ 


\section{WILEY-VCH}

and $200 \mathrm{~nm}$ for SEM images i, ii and iii respectively. The scale bar for (iii)'s inset image is $500 \mathrm{~nm}$. See Experimental Section for full fabrication details.

(a)i.
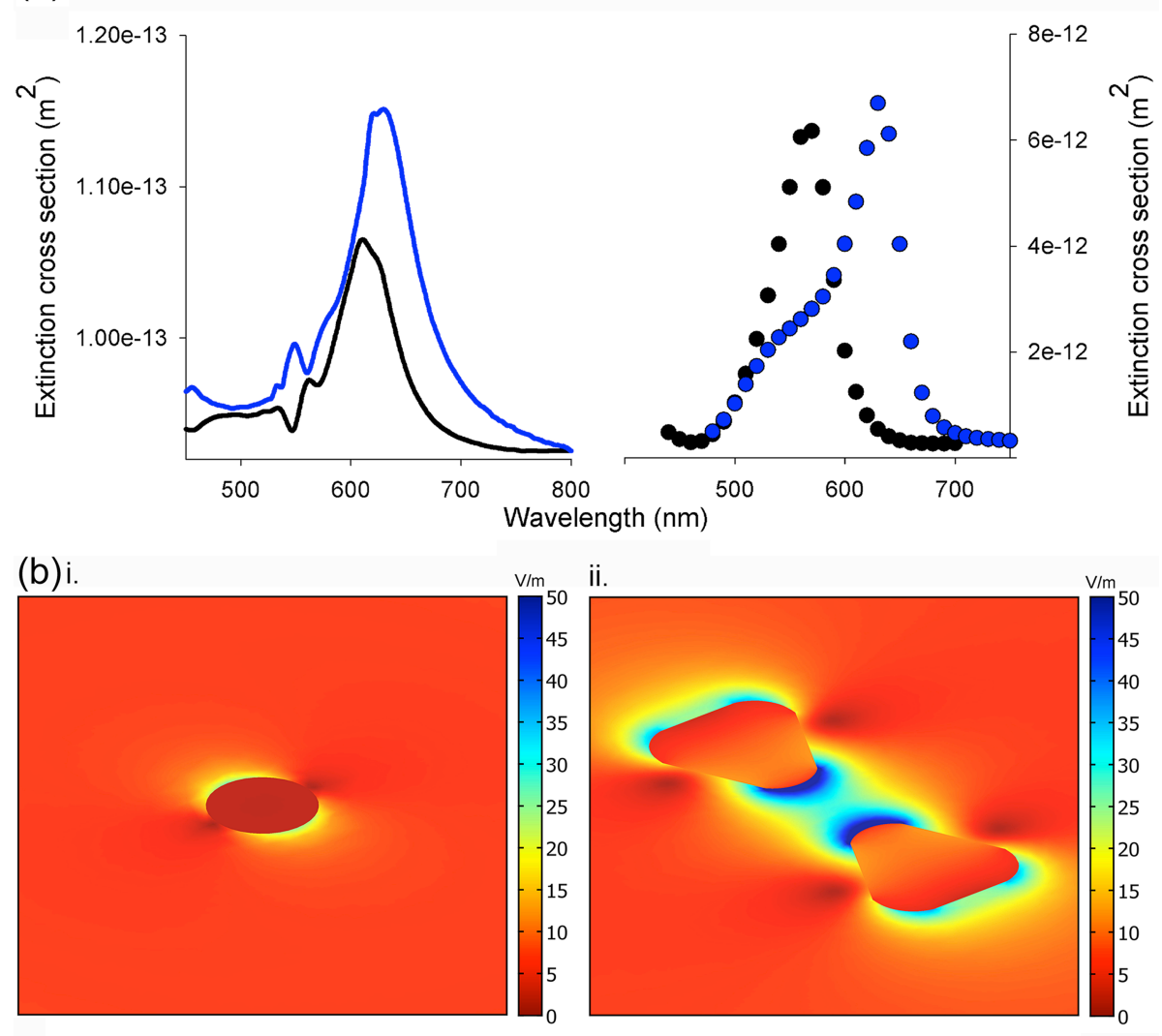

iii.

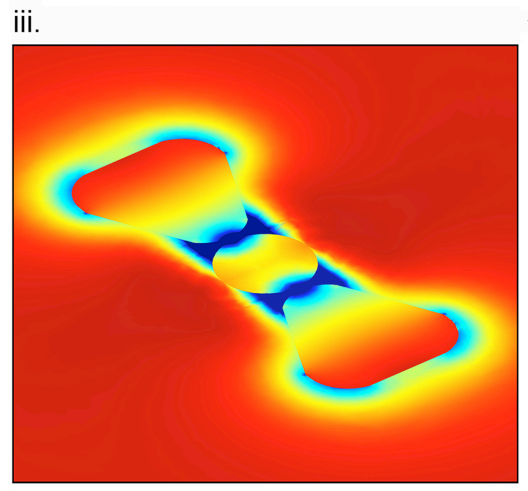

ii.

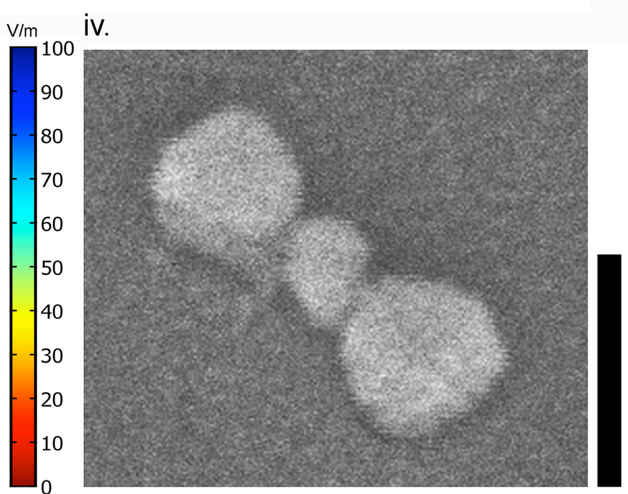

Figure 3. Plasmonic characterization of the bowtie devices before and after completion with a DNA nanoparticle. 3(a) Experimental (i) and simulated (ii) absorbance spectra detailing the LSPR peak before (black) and after (blue) hybridization. The arrays produced for spectral analysis were $700 \mu \mathrm{m}$ squares and had a periodicity of $300 \mathrm{~nm}$. (b) Simulated electric field enhancements, at resonance, of an isolated nanoparticle (i), an incomplete bowtie (ii), and a 


\section{WILEY-VCH}

bowtie coupled to a nanoparticle (iii). The side-bar for each simulation represents the normalized electric-field enhancement factor given an incident field value of $1 \mathrm{~V} / \mathrm{m}$. The range depicted by these images do not necessarily represent the largest enhancements experienced by the structures, but were chosen to best illustrate the field distribution and intensity gradient. Maximum enhancements of 27, 59 and $>1700$ were experienced by the isolated nanoparticle, incomplete and complete bowties respectively. For the bowties the maximum fields were experienced at the tips of the structure's prisms 3(b)(iv) shows an SEM of the completed structure. The length of the scale bar is $100 \mathrm{~nm}$. 


\section{WILEY-VCH}
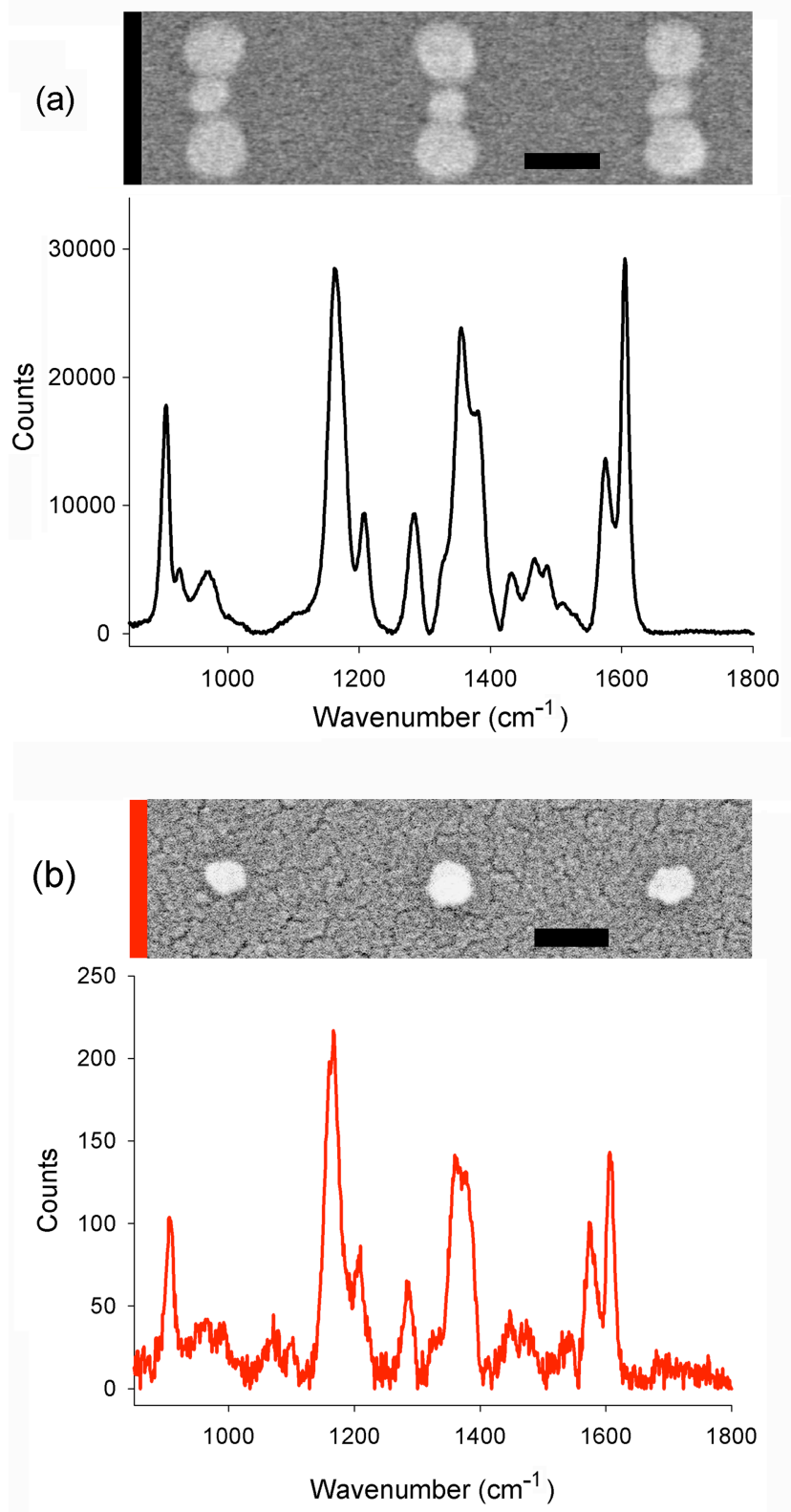

Figure 4. SERS spectra comparison of plasmonic bowtie containing single DNAnanoparticles and isolated nanoparticles (a) SERS spectra and SEM of completed devices (b) SERS spectra and SEM of isolated nanoparticles. The scale bars in each SEM represent 100 $\mathrm{nm}$. The periodicity for both arrays was $300 \mathrm{~nm}$. The acquisition time for each spectra was 5 seconds. The Raman scattered output from the coupled devices is 2 orders of magnitude larger than that of the scattered light collected from the isolated particles. 


\section{WILEY-VCH}

(a)
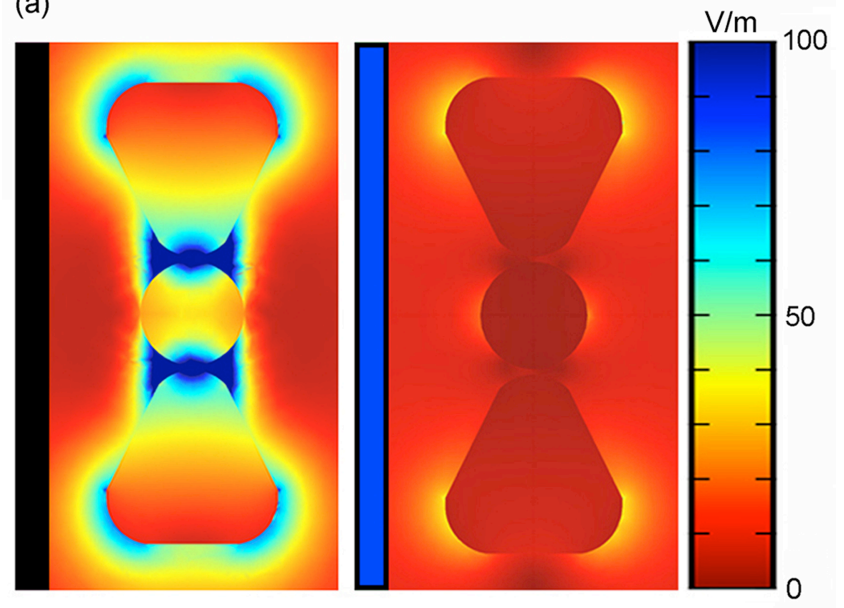

(b)

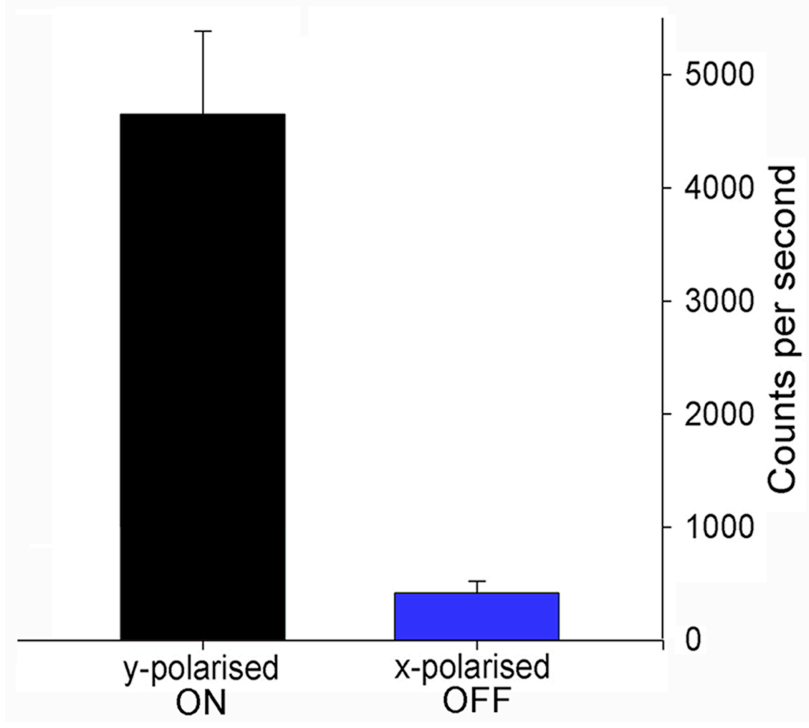

Figure 5. Polarization dependent Raman outputs. (a) FEM simulations of the device excited with the input polarized along the $\mathrm{y}$-axis (left) and the $\mathrm{x}$-axis (right). The side-bar for each simulation represents the normalized electric-field enhancement factor given an incident field value of $1 \mathrm{~V} / \mathrm{m}$. (b) Comparison of average SERS intensity, with standard deviation, for a 300 $\mathrm{nm}$ period bowtie at these two input conditions. 8 measurements were taken for each input. 

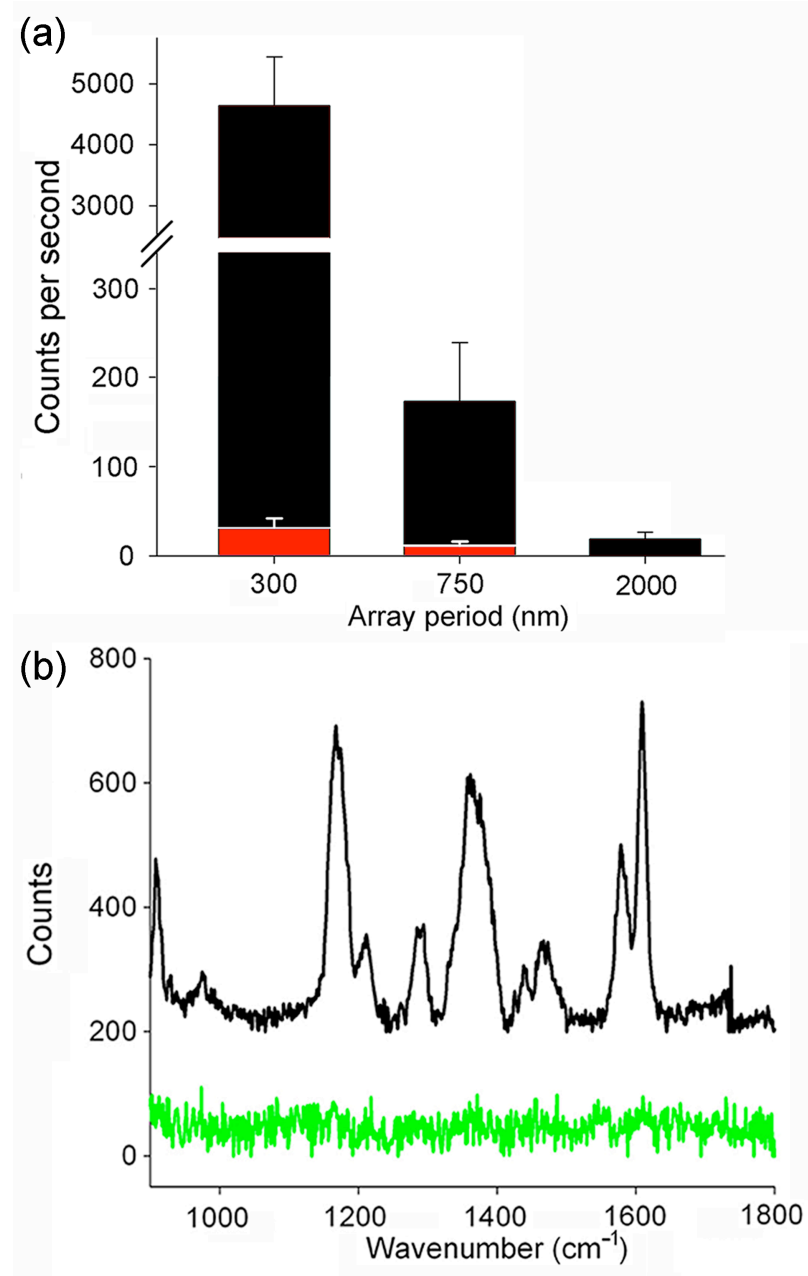

Figure 6. Detecting single nanoparticle binding events. (a) Comparison of SERS intensity, with standard deviation, from the $1605 \mathrm{~cm}^{-1}$ band of malachite green, for different densities of isolated nanoparticles (red bars) and nanoparticles situated in a completed circuit (black bars). 8 measurements were taken for each data point. Acquisition times were 5 seconds for the 300 $\mathrm{nm}$ period array and 20 seconds for the 750 and $2000 \mathrm{~nm}$ period arrays. Data is presented in counts per second for simplicity of viewing. (b) SERS spectra of a single isolated nanoparticle (green) and a single nanoparticle bound within a plasmonic bowtie via DNA hybridization. The acquisition time was 20 seconds in each case. 


\section{WILEY-VCH}

Single DNA-nanoparticle binding is used to couple the plasmonic fields of nanophotonic bowtie dimers. The binding event is engineered to tune the resonance peak of the bowtie to correspond to a $633 \mathrm{~nm}$ laser. Surface enhanced Raman spectroscopy is performed on an individual bowtie, showing that a single DNA-nanoparticle binding event can be recorded.

Plasmonics, Surface Enhanced Raman Spectroscopy, DNA, Biosensor, Molecular assembly

A.W. Clark*, D.G. Thompson, D. Graham, J.M. Cooper

Engineering DNA binding sites to assemble and tune plasmonic nanostructures

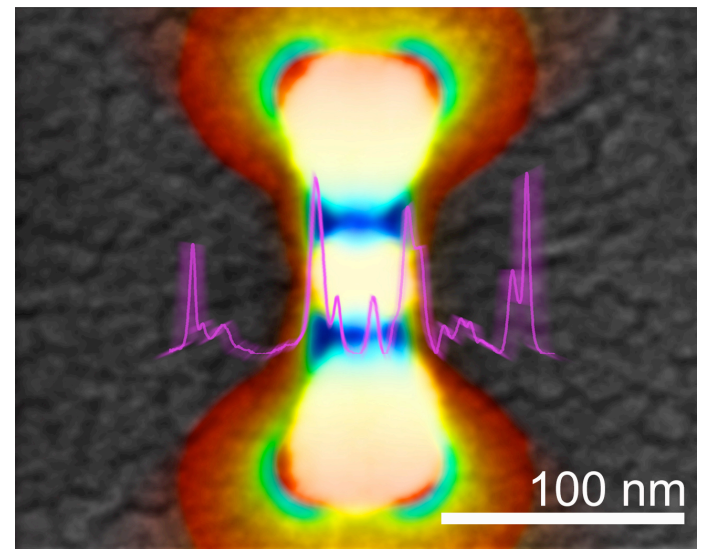

